\title{
First single-shot and non-intercepting longitudinal bunch diagnostics for comb-like beam by means of Electro-Optic Sampling
}

\author{
R. Pompili ${ }^{\mathrm{a}, \mathrm{b}, *}$, A. Cianchi ${ }^{\mathrm{b}}$, D. Alesini ${ }^{\mathrm{a}}$, M.P. Anania ${ }^{\mathrm{a}}$, A. Bacci ${ }^{\mathrm{c}}$, M. Bellaveglia ${ }^{\mathrm{a}}$, \\ M. Castellano a, E. Chiadroni ${ }^{\text {a }}$, D. Di Giovenale ${ }^{a}$, G. Di Pirro ${ }^{a}$, G. Gatti ${ }^{a}$, F. Giorgianni ${ }^{\mathrm{d}}$, \\ M. Ferrario ${ }^{\text {a }}$, S. Lupi ${ }^{\text {d }}$, F. Massimo ${ }^{\text {d }}$, A. Mostacci ${ }^{a}$, A.R. Rossi ${ }^{\text {c }}$, C. Vaccarezza ${ }^{\text {a }}$, F. Villa ${ }^{\text {a }}$ \\ a INFN-LNF, Via E. Fermi 40, 00044 Frascati, Rome, Italy \\ ${ }^{\mathrm{b}}$ Università di Roma "Tor Vergata", Physics Department, Via della Ricerca Scientifica 1, 00133 Rome, Italy \\ ' INFN-Milano, Via Celoria 16, 20133 Milan, Italy \\ ${ }^{\mathrm{d}}$ Università di Roma “Sapienza", Physics Department, Via Aldo Moro 2, 00185 Rome, Italy
}

\section{A R T I C L E I N F O}

Available online 26 October 2013

Keywords:

Plasma

Diagnostics

Electro-optic

EOS

\begin{abstract}
A B S T R A C T
At SPARC-LAB, we have installed an Electro-Optic Sampling (EOS) experiment for single shot, nondestructive measurements of the longitudinal distribution charge of individual electron bunches. The profile of the electron bunch field is electro-optically encoded into a Ti:Sa laser, having $130 \mathrm{fs}(\mathrm{rms})$ pulse length, directly derived from the photocathode's laser. The bunch profile information is spatially retrieved, i.e., the laser crosses with an angle of $30^{\circ}$ with respect to the normal to the surface of EO crystal (ZnTe, GaP) and the bunch longitudinal profile is mapped into the laser's transverse profile. In particular, we used the EOS for a single-shot direct visualization of the time profile of a comb-like electron beam, consisting of two bunches, about $100 \mathrm{fs}$ (rms) long, sub-picosecond spaced with a total charge of $160 \mathrm{pC}$. The electro-optic measurements (done with both ZnTe and GaP crystals) have been validated with both RF Deflector (RFD) and Michelson interferometer measurements.
\end{abstract}

(c) 2013 Elsevier B.V. All rights reserved.

\section{Introduction}

Single-shot and non-intercepting bunch length measurements with 50 fs order resolution are of high interest for future plasmabased accelerators, in order to monitor the beams to be injected in the plasma. In particular, the particle-driven wakefield acceleration (PWFA) require multi-bunches schemes where two or more subsequent bunches, sub-100 fs long and sub-picosecond spaced, can enhance the plasma transformer ratio $R$ and generate the proper accelerating field [1] able to accelerate the last one with low energy spread [2].

The technique of electro-optical sampling (EOS) [3] provides the possibility of measuring the longitudinal charge distribution by means of nonlinear crystals placed near the moving electron beams and it is able to reach high temporal resolutions, determined by the width of the optical laser pulse and the EO crystal length. The working principle is based on the induced birefringence in a nonlinear crystal (like ZnTe and GaP) by the high electric fields of the relativistic electron bunch (with temporal profile $\left.E_{\text {bunch }}(t)\right)$, which propagate in the crystal like a THz-field [4]

\footnotetext{
* Corresponding author at: INFN-LNF, Via E. Fermi 40, 00044 Frascati, Rome, Italy. Tel.: + 393202835718

E-mail address: riccardo.pompili@lnf.infn.it (R. Pompili).
}

(see Fig. 1). Since the crystal becomes anisotropic (biaxial), the electric field of a polarized laser passing in the crystal is decomposed along the two optical axes, with characteristic refractive indices $n_{i}=n_{1}, n_{2}$. Because the two components travel at different velocities $v_{i}=c / n_{i}$, at the end of the crystal their relative phase delay $\Gamma$ is

$\Gamma(t)=\frac{\omega d}{c}\left(n_{1}-n_{2}\right) \propto E_{\text {bunch }}(t)$

where $\omega$ is the laser's pulse frequency and $d$ is the crystal thickness. Therefore the time information contained in $\Gamma(t)$ is a replica of $E_{\text {bunch }}(t)$.

At the SPARC-LAB facility [5] (see Fig. 2) a Ti:Sa IR laser ( $\lambda=800 \mathrm{~nm}, 130 \mathrm{fs}$ pulse length, rms) is used to sample the birefringence which is induced in the nonlinear optical crystal by the co-moving electric field of a $110 \mathrm{MeV}$ electron bunch. The laser is directly derived from the photocathode's one, resulting in a natural synchronization with the electron beam, having a repetition rate of $10 \mathrm{~Hz}$. The initial linear polarization of the laser pulse is converted into a slightly elliptical polarization which is then converted into an intensity modulation by placing a polarizer after the crystal, with its polarization axis rotated by $90^{\circ}$ with respect to the initial laser polarization. To encode the bunch longitudinal profile into the laser, we used the spatially encoding EOS technique [6], in which the laser crosses the nonlinear crystal with an 


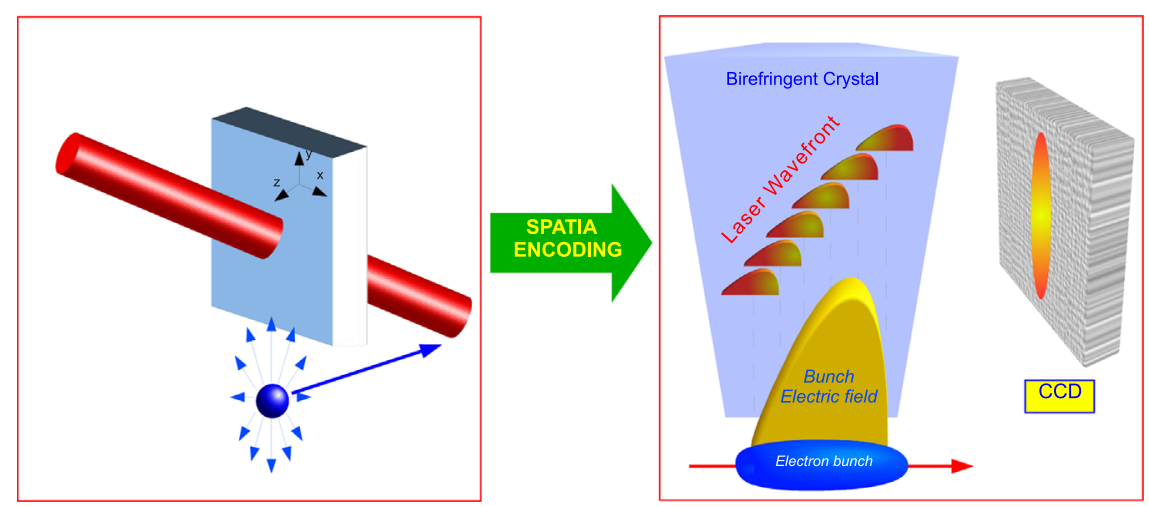

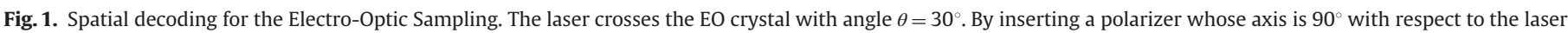
linear polarization, the longitudinal bunch profile is directly retrieved on the CCD.

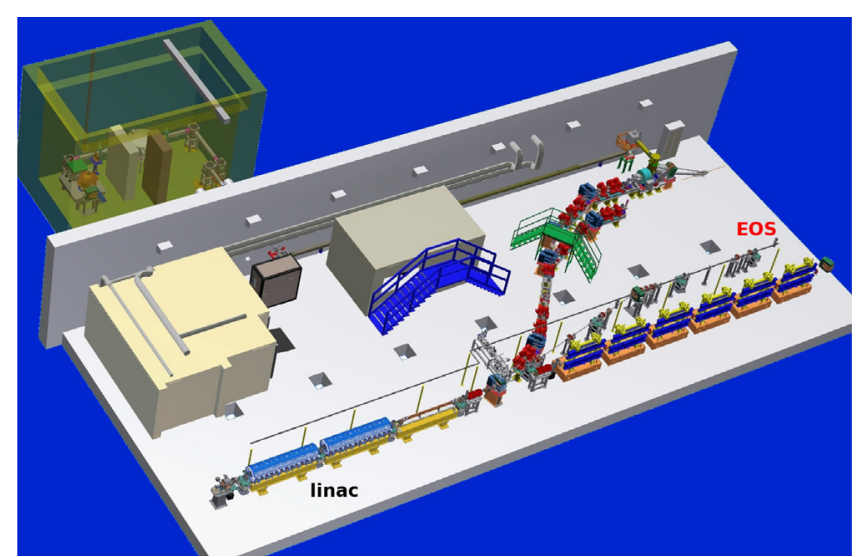

Fig. 2. SPARC-LAB layout. The EOS station is located at the end of the 2nd beamline.

angle of $\theta=30^{\circ}$ (see Fig. 1). In such a way, being $x$ the spatial coordinate along the laser transverse profile and $t$ the time coordinate for the longitudinal bunch profile, we have

$t=\frac{x}{c} \tan \theta$

where $c$ is the vacuum speed of the light. From Eq. (2), the total time window $\Delta t$ is directly proportional to the laser's spot diameter $d$, i.e., $\Delta t=(d / c) \tan \theta$. In the presented measure, a $5 \mathrm{~mm}$ wide laser has been used, resulting in a time window of about 10 ps.

Previous accelerator-related EOS experiments have been carried out at FELIX [7], DESY [8] and SLAC [9], in each of which the EOS has been tested on a single (short or long) electron bunch. Here we report the recent results achieved at SPARC-LAB measuring, for the first time, the longitudinal profile of comb-like beams, consisting of two bunches, about $100 \mathrm{fs}$ (rms) long, sub-ps spaced with a total charge up to $160 \mathrm{pC}$.

\section{Two bunches comb-like beam at SPARC-LAB}

The comb-like beam consists of two approximately equal electron bunch, generated by properly shaping trains of UV laser pulses illuminating the metallic photo-cathode in the RF gun [10]. A diagnostics transfer line allows to fully characterize the accelerated beam by measuring transverse emittance [11] and the longitudinal profile through a Radio-Frequency Deflector (RFD) [12], located at the linac exit. Fig. 3(a) and (b) shows, respectively, the longitudinal phase space and the corresponding longitudinal profile for a $160 \mathrm{pC}$ total charge beam. The two consecutive (a)

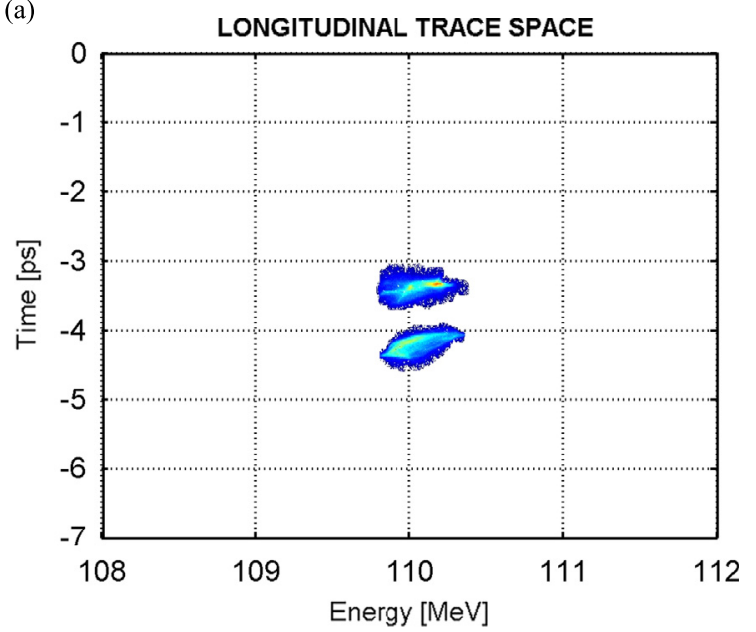

(b)

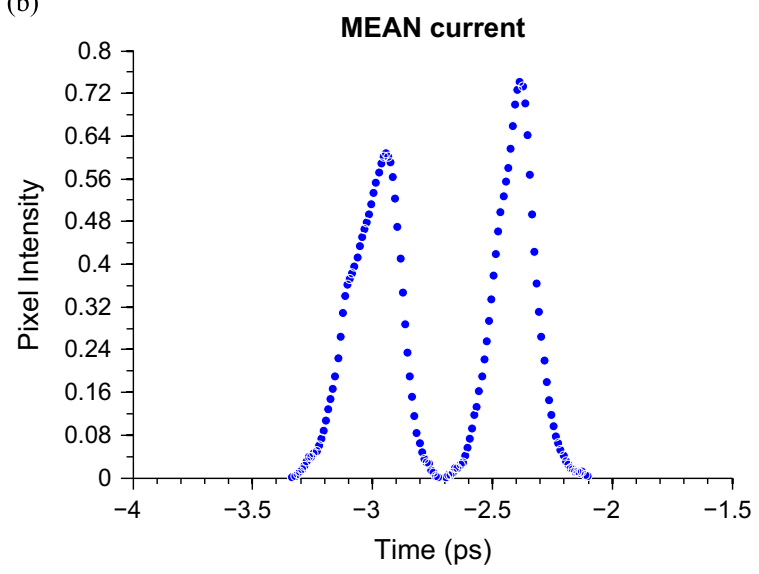

Fig. 3. (a) Longitudinal phase space retrieved by using a RF Deflector (time) and a bending magnet (energy) of a comb beam consisting of two consecutive bunches with a mean energy of $110.0 \pm 0.1 \mathrm{MeV}$, with a total charge of $160 \mathrm{pC}$ (77 pC and $83 \mathrm{pC}$ for each bunch). (b) Projected profile (along the time axis), resulting in bunch lengths of $100 \pm 12$ fs and $70 \pm 17$ fs (both rms), separated by $846 \pm 15$ fs.

bunches have lengths of $100 \pm 12 \mathrm{fs}$ and $70 \pm 17 \mathrm{fs}$ (rms), with respective charges of $77 \pm 6 \mathrm{pC}$ and $83 \pm 7 \mathrm{pC}$, separated by $846 \pm 15$ fs with a mean energy of $110.0 \pm 0.1 \mathrm{MeV}$.

Because the EOS station is located at the end of a dogleg transfer line, we have to take care to the fact that the longitudinal phase space evolution in the dogleg is dominated by nonlinearities given by high order chromatic terms $[13,14]$. In order to evaluate the bunch properties after this line, a Michelson interferometer, analyzing the coherent transition radiation (CTR) from an 

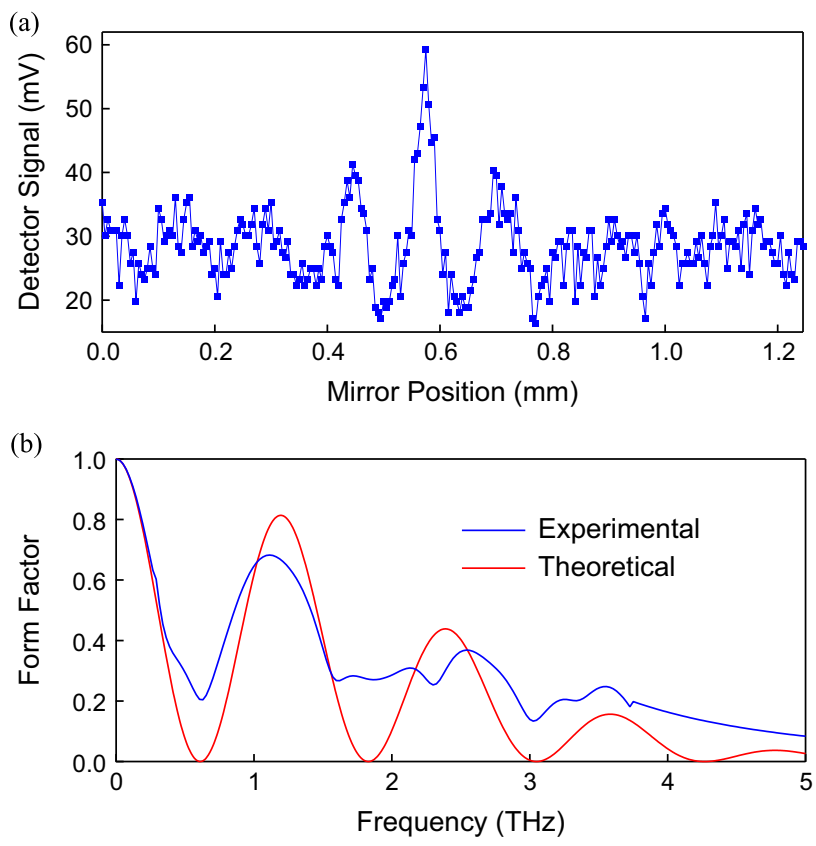

Fig. 4. (a) Interferogram obtained by moving the translational stage at steps of $10 \mu \mathrm{m}$ and (b) retrieved form factor of the comb beam measured with the Michelson Interferometer after the dogleg line. The calculated bunch distance is $830 \pm 20 \mathrm{fs}$ while the upper limit for the bunch lengths is fixed at $\sim 150 \mathrm{fs}$ (rms).

aluminium coated silicon screen, has been installed at the end of the dogleg line just before the EOS station; CTR is produced when a relativistic charged particle crosses the interface between two media of different dielectric properties (vacuum and aluminium, in our case) and its frequency spectrum follows the electron bunch one. ${ }^{1}$ The interferometer consists in a $12 \mu \mathrm{m}$ mylar sheet that splits the CTR radiation into two arms, one of which can be changed in length by using a motorized translational stage. By recombining the two radiations on a broadband $(0.5-30 \mathrm{THz})$ pyroelectric detector, the overall intensity modulation (sampled for each position of the translational stage by steps of $10 \mu \mathrm{m}$ ) is used to make the interferogram shown in Fig. 4(a) and determine the corresponding CTR form factor (Fig. 4(b)), i.e., the power spectrum of the electron beam [15]. Since from the production to the detection point the CTR crosses a quartz window whose characteristic cut-off frequency is about $3.8 \mathrm{THz}$, the Michelson interferometer is able to reproduce bunch spectra up to this frequency. In our measurement we obtained a bunch distance of $830 \pm 20 \mathrm{fs}$ and $150 \mathrm{fs}$ (rms) as the upper limit of the bunch lengths, results that are comparable with the RFD data.

\section{EOS experimental setup}

In our experiment we used ZnTe and GaP with thicknesses of $500 \mu \mathrm{m}$ and $400 \mu \mathrm{m}$, respectively, both cut in the (110) plane (Ingcrys Laser Systems, UK); therefore, in order to have a net EO effect, their $[-1,1,0]$ axes have to be parallel to both the bunch and the laser electric fields [8]. By doing so, the induced phase delay of Eq. (1) becomes

$\Gamma(t)=\frac{\omega n_{0}^{3} r_{41} E_{\text {bunch }}(t)}{c} d$

being $r_{41}$ the electro-optic coefficient of the crystal.

${ }^{1}$ Except a strong suppression of the low frequencies side, due to the effect of considering the finite size of the metal target and far-field approximation not completely fulfilled [14]. (a)

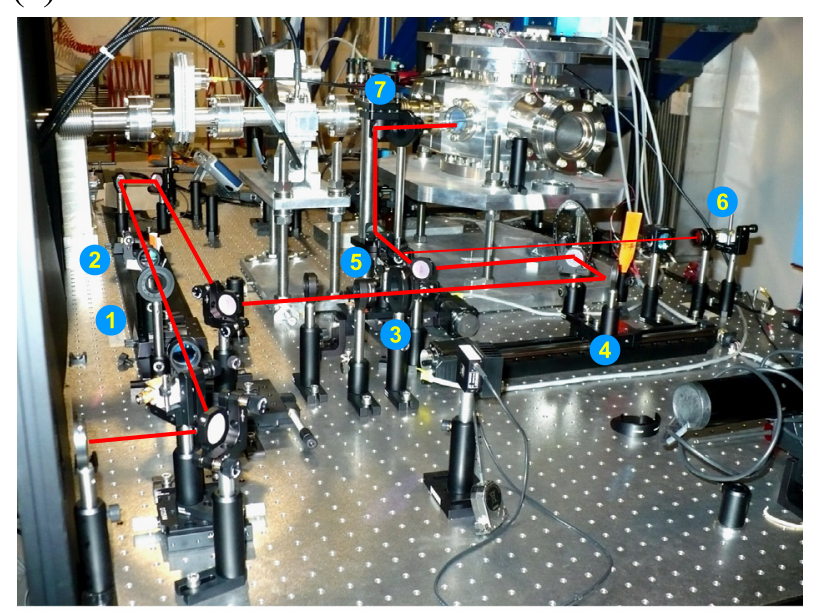

(b)

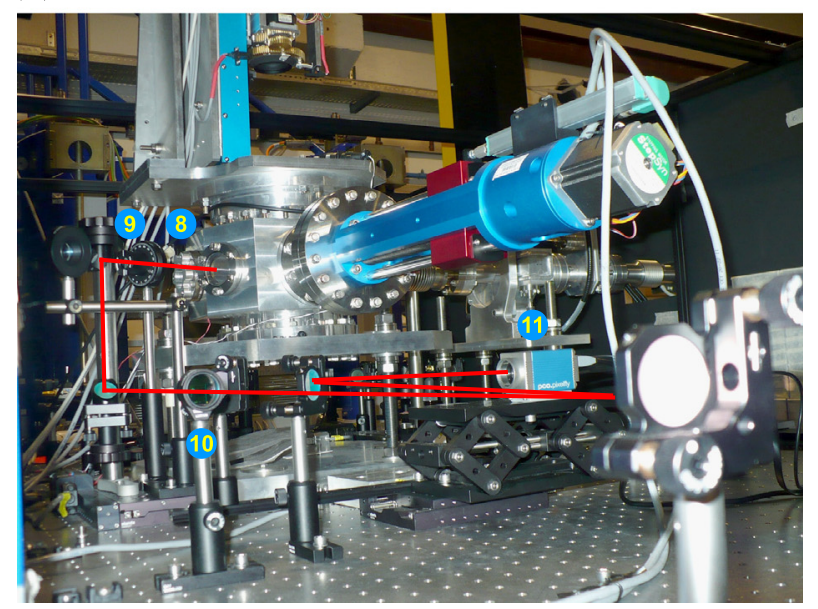

Fig. 5. (a) Optical layout before the EOS vacuum chamber. The IR laser passes through a telescopic system (1) and a polarizing beam splitter (2). The resulting p-polarized beam is sent to a half-wave plate (3), then passes through an optical delay line (4) and a non-polarizing beam splitter, sending half of the beam to the synchronization photodiode (6) and the other half to a Glan-laser polarizer (7). (b) Optical layout after the EOS vacuum chamber. The laser passes a quarter-wave plate (8) and another Glan-laser polarizer (9), crossed respect to the previous one. A lens (10) images the laser on the CCD camera (11).

The EOS optical setup is shown in Fig. 5(a) and (b). The EO crystals are positioned so that their $[-1,1,0]$ axis is properly oriented, i.e., parallel to the bunch electric field. The crystals are mounted on a remotely controlled actuator, which also holds OTR and Ce:YAG screens to monitor the electron beam position. By knowing such a position, it is possible to move the actuator in order to approach the crystals to the beam up to several microns. The IR laser's energy is adjusted by simply rotating an half-wave plate placed between two polarizers. In the experiment, the laser energy was $50 \mathrm{~nJ}$. The synchronization between the laser itself and the electron beam is automatically achieved since the laser is the same that is used for the photocathode. A fine adjustment is however possible by using an optical delay line installed prior to the EO crystal. In order to reach the synchronization between the travelling electron bunch and the IR laser pulse, two arrival time monitor devices are needed. For the laser we used a fast rise time (30 ps) G4176 photodiode by Hamamatsu ${ }^{\mathrm{TM}}$, while to retrieve the electron bunch arrival time we looked directly to the $5 \mathrm{GHz}$ signals coming from a Cavity BPM installed prior to the EOS station. Since both devices have broadband outputs, the signals are analyzed by 


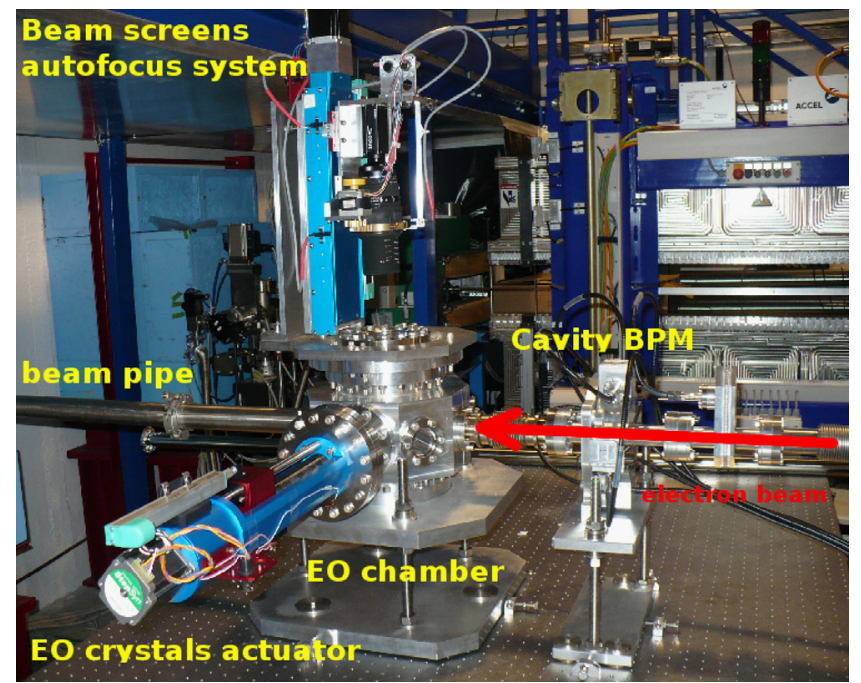

Fig. 6. EOS experimental station. A cavity BPM is placed before the EOS chamber to monitor the bunch arrival time. The EOS chamber consists in two actuators, one for the EO crystals and the beam screens and the other to move the focus and the position of the top camera looking at these screens.

a $20 \mathrm{GHz}$ scope by Tektronix ${ }^{\mathrm{TM}}$. The EOS station with the Cavity BPM is shown in Fig. 6.

The Glan-laser polarizer located before the input window produces a horizontally polarized laser, necessary to have its electric field parallel to the $[-1,1,0]$ axis of the crystal. After the crystal, another Glan-laser polarizer (crossed with respect to the previous one) is used, preceded by a quarter-wave plate useful to reduce the residual birefringence (i.e., not coming from the EO effect) mainly due to the induced stress caused by the high vacuum inside the chamber in the input and exit fused silica windows. With such a polarizer's setup, the output signal detected by the CCD is

$I_{\text {det }}=I_{\text {laser }} \sin ^{2}\left(\frac{\Gamma}{2}\right) \approx I_{\text {laser }}\left(\frac{\omega n_{0}^{3} r_{41} E_{\text {bunch }}(t)}{c} d\right)^{2}$

where $I_{\text {det }}$ and $I_{\text {laser }}$ are, respectively, the signal and laser's intensity and $\Gamma$ is the relative phase delay induced by the electro-optic effect. Eq. (4) shows that the output signal is proportional to the square of the field. At the end of the setup, a PixelFly USB CCD camera by $\mathrm{PCO}^{\mathrm{TM}}$, triggered to the bunch emission rate at $10 \mathrm{~Hz}$, is used to retrieve the EO signals by looking at the laser's intensity modulation. With a pixel dimension of $6.45 \mu \mathrm{m}$, and considering an optical magnification $M=0.69$ of the signal on the $C C D$, according to Eq. (2) the pixel time resolution is about $18 \mathrm{fs}$.

\section{EOS results}

Two typical single-shot EO measurements are shown in Figs. 7(a) and 8(a), showing the output signal from ZnTe and GaP, respectively; the time direction (i.e., the signal longitudinal profile) is marked by a white arrow. Because the laser's intensity instability is about $8 \%$, for a reconstruction of the bunch profile, several screenshots with the electron beam turned off are acquired in order to have an averaged background screen-shot to subtract to the signal's one; all background traces were taken with the same laser and CCD camera settings that were used for the EO measurements.

Fig. 7(b) shows a single shot EO signal after background subtraction for ZnTe. A Gaussian fit has been calculated on the experimental data, showing a sigma value of $434 \pm 9 \mathrm{fs}$ (rms) for the first bunch and $331 \pm 6 \mathrm{fs}$ (rms) for the second one, with a distance between the two peaks equal to $921 \pm 7$ fs. To take into (a)

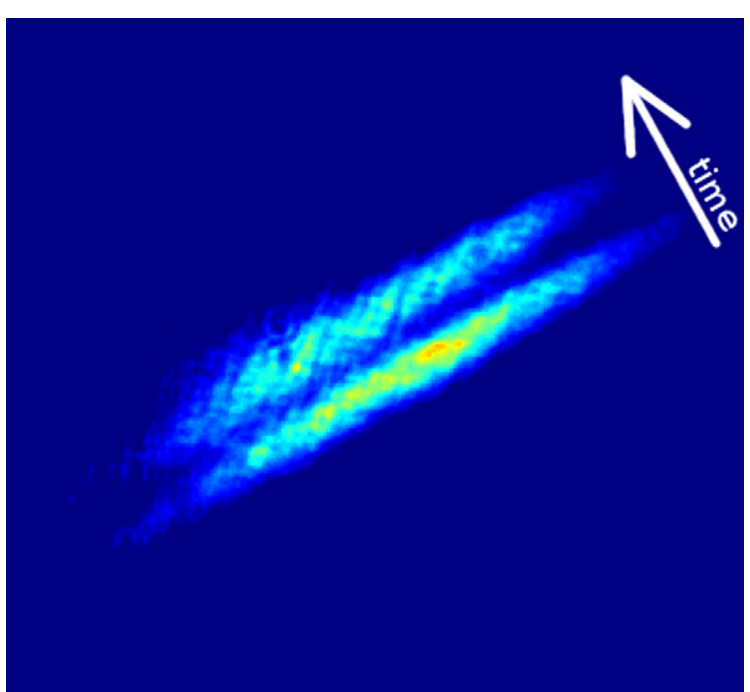

(b)

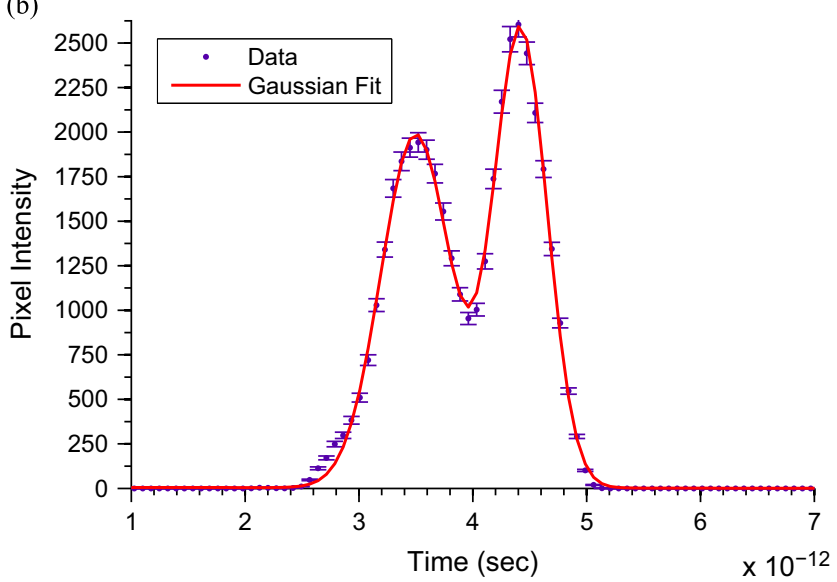

Fig. 7. (a) EOS single screen-shot for the comb beam retrieved by using the $500 \mu \mathrm{m}$ ZnTe crystal. The time direction is marked by the white arrow. (b) Projected profile (along the time axis) with Gaussian fit, with calculated sigma for the two main peaks of $434 \pm 9$ fs and $331 \pm 6$ fs (rms), separated by $921 \pm 7 \mathrm{fs}$. By making an average of 100 consecutive shots, the average lengths of the two bunches are $444 \pm 46 \mathrm{fs}$ and $340 \pm 14 \mathrm{fs}$ (rms) with an average distance of $932 \pm 27 \mathrm{fs}$. The signal (and the Gaussian fit) is proportional to the square of $E_{\text {bunch }}$ (Eq. (4)).

account possible fluctuations on the bunch lengths, ${ }^{2}$ an average of 100 consecutive shoots has been calculated, showing mean lengths of $444 \pm 46$ fs and $340 \pm 14$ fs (rms) with a mean distance of $932 \pm 27$ fs. The same computations have been done for the GaP crystal and the result is shown in Fig. 8(b); the Gaussian fit calculated in the shown screen-shot of Fig. 8(a) reports sigma values of $329 \pm 18 \mathrm{fs}$ and $336 \pm 11 \mathrm{fs}$ (rms) for the two bunch lengths and a distance of $931 \pm 12$ fs between the two peaks in the trace. By making an average of 100 consecutive shots, the average lengths of the two bunches are $461 \pm 68$ fs and $346 \pm 27 \mathrm{fs}$ (rms) with a mean distance of $864 \pm 58$ fs.

The previous results obtained with a $500 \mu \mathrm{m}$ ZnTe and a $400 \mu \mathrm{m}$ GaP show that the obtained bunch distance is the same (inside the errors) for both crystals and its value is compatible with the RFD data by assuming the overall transport in the dogleg line [14], while the calculated bunch lengths are quite larger. This is an expected behaviour, first of all because the laser pulse length used in the

${ }^{2}$ E.g., due to the time jitter between the RF and the photocathode's laser and $\mathrm{RF}$ peak power instabilities. 
(a)

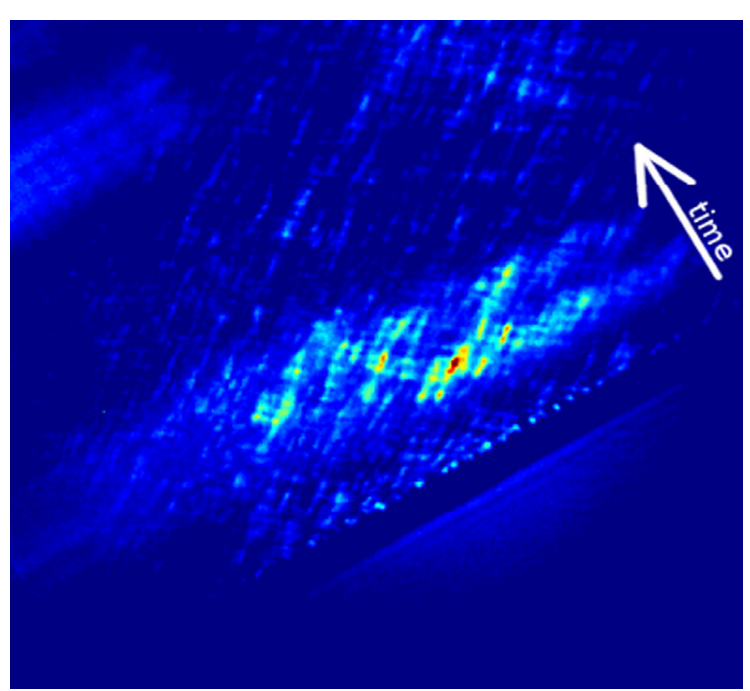

(b)

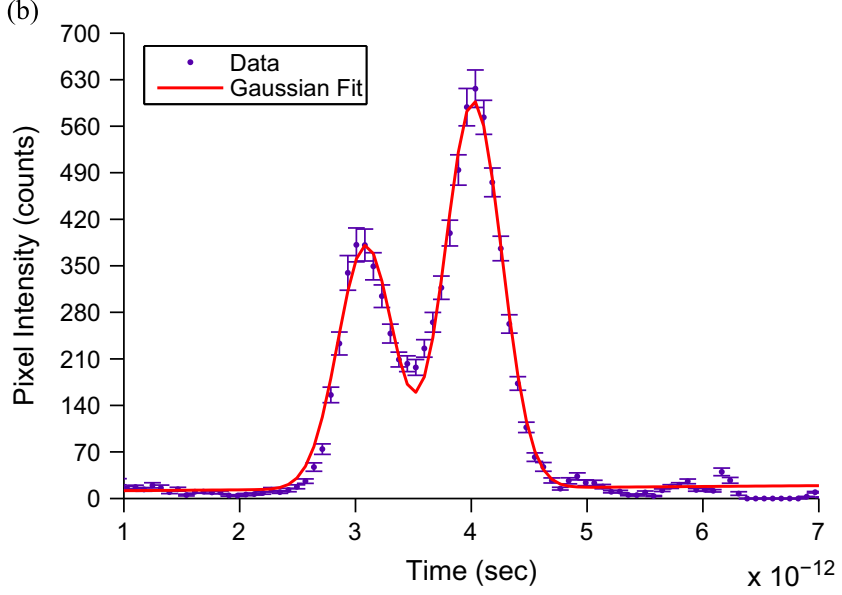

Fig. 8. (a) EOS single screen-shot for the comb beam retrieved by using the $400 \mu \mathrm{m}$ GaP crystal. The time direction is marked by the white arrow. (b) Projected profile (along the time axis) with Gaussian fit, with calculated sigma for the two main peaks of $329 \pm 18 \mathrm{fs}$ and $336 \pm 11 \mathrm{fs}$ (rms), separated by $931 \pm 12 \mathrm{fs}$. By making an average of 100 consecutive shots, the average lengths of the two bunches are $461 \pm 68 \mathrm{fs}$ and $346 \pm 27 \mathrm{fs}$ (rms) with an average distance of $864 \pm 58 \mathrm{fs}$. The signal (and the Gaussian fit) is proportional to the square of $E_{\text {bunch }}$ (Eq. (4)).

experiment is longer than the electron bunch lengths. Then, one has to take into account the velocity mismatch (between the propagating laser and the $\mathrm{THz}$ pulses) and the phonon resonances (located at 5.3 THz for ZnTe and $10.98 \mathrm{THz}$ for GaP) of the two crystals. Fig. 9 (a) and (b) shows a simulation for the expected bunch lengths reconstructed by the EOS technique with different values of the ZnTe and GaP thickness, respectively. The broadening is proportional to the crystal's thickness since the different frequencies of the $\mathrm{THz}$ pulse travel at different velocities ${ }^{3}$ with respect to each other and with respect to the laser; because of this slippage, the THz pulse "appears" longer to the laser. Moreover, the absorption and refractive indices of the crystals grow rapidly for frequencies approaching the phonon's resonances, therefore higher frequencies (corresponding to shorter bunches) propagate slowly and the velocity mismatch increases. In conclusion, currently the crystal thickness and the laser pulse length fix the EOS temporal resolution that can be improved by reducing the thickness itself (at the price of a lower EOS signal intensity) while compressing the laser up to its transform limit (about $50 \mathrm{fs}$ ). In this sense, Fig. 9(b) shows that $100 \mu \mathrm{m}$-order GaP crystals are able to

\footnotetext{
${ }^{3}$ Because the crystal refractive index is a function of the frequency.
}

(a)

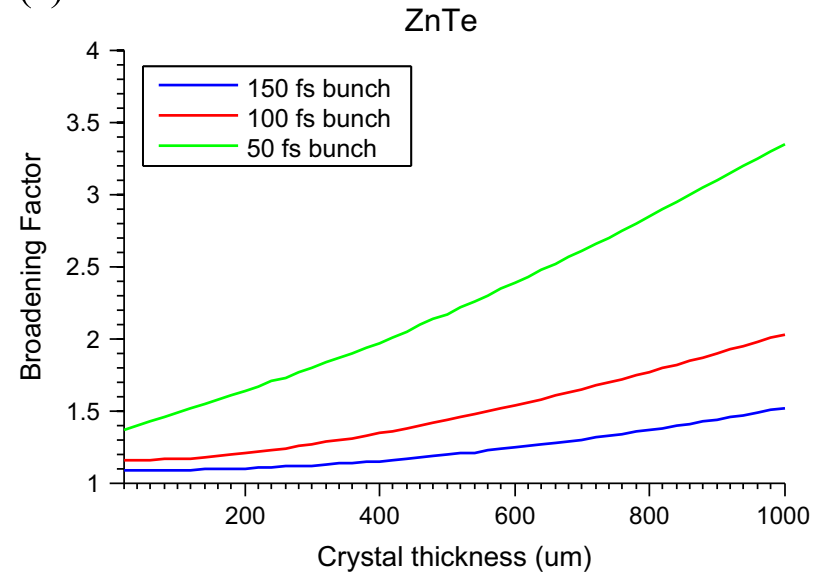

(b)

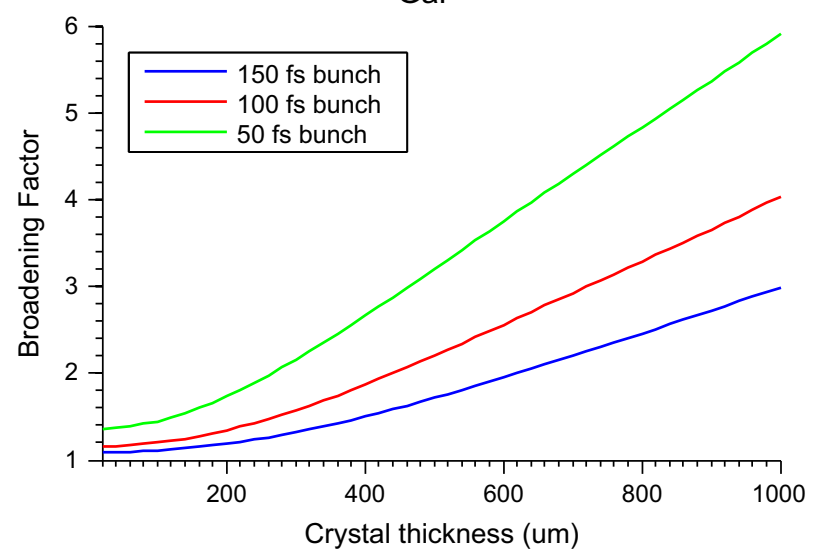

Fig. 9. Expected (theoretical) pulse broadening in $\mathrm{ZnTe}$ (a) and GaP (b) for different crystal's thicknesses. The rms length of the electron bunches $(150,100$ and $50 \mathrm{fs})$ are shown in different coloured lines. (For interpretation of the references to colour in this figure caption, the reader is referred to the web version of this paper.)

measure bunch length of the order of $50 \mathrm{fs}$ (rms), because in this crystal the phonon resonance is localized at higher frequencies with respect to ZnTe.

\section{Conclusion and outlook}

In this contribution, we used for the first time the electro-optic sampling (EOS) technique to measure the longitudinal profile of a comb-like beam consisting of two bunches. The response of the electro-optic crystals, ZnTe and GaP in our case, is the dominant temporal limitation in these measurements. Simultaneous RFD and interferometric measurements show a good agreement with EOS data regarding the distance between the two bunches, while the calculated EOS single bunch lengths appear greater due to the velocity mismatch between the $\mathrm{THz}$ and laser pulses inside the crystal. With GaP thicknesses of the order of $100 \mu \mathrm{m}$ a near crossed polarizer setup [8] should be used in order to increase the signal on the camera while having a signal dependence on the beam electric field almost linear. By using thinner crystals with a shorter laser pulse length, the time resolution can reach about 50 fs. The non-invasive property of the electro-optic measurement will allow it to be used as a diagnostic tool for electron beams to be injected in future PWFA accelerators. 


\section{Acknowledgements}

This work has been partially supported by the EU Commission in the Seventh Framework Program, Grant Agreement 312453EUCARD-2 and the Italian Minister of Research in the framework of FIRB - Fondo per gli Investimenti della Ricerca di Base, Project no. RBFR12NK5K.

\section{References}

[1] E. Kallos, Plasma wakefield accelerators using multiple electron bunches (Ph.D. thesis), Faculty of the Graduate School, University of Southern California, August 2008.

[2] T. Katsouleas, C. Joshi, J.M. Dawson, F.F. Chen, C. Clayton, W.B. Mori, C. Darrow, D. Umstadter, Plasma accelerators, in: American Institute of Physics Conference Series, vol. 130, 1985, pp. 63-98. http://dx.doi.org/10.1063/1.35293.

[3] S. Casalbuoni, H. Schlarb, B. Schmidt, B. Steffen, P. Schmuser, A. Winter Numerical studies on the electro-optic sampling of relativistic electron bunches, in: Proceedings of the IEEE Particle Accelerator Conference, PAC, 2005, pp. 3070-3072.

[4] S. Jamison, G. Berden, A.M. MacLeod, D. Jaroszynski, B. Redlich, A. Van der Meer, W. Gillespie, Nucl. Instrum. Methods Phys. Res. Sect. A: Accel. Spectrom. Detectors Assoc. Equip. 557 (1) (2006) 305.

[5] D. Alesini, et al., Nucl. Instrum. Methods Phys. Res. Sect. A: Accel. Spectrom. Detectors Assoc. Equip. 507 (1-2) (2003) 345 http://dx.doi.org/10.1016 S0168-9002(03)00943-4.
[6] A.L. Cavalieri, D. Fritz, S. Lee, P. Bucksbaum, D. Reis, J. Rudati, D. Mills, P. Fuoss, G. Stephenson, C. Kao, et al., Phys. Rev. Lett. 94 (11) (2005) 114801.

[7] G. Berden, S.P. Jamison, A.M. MacLeod, W. Gillespie, B. Redlich, A. van der Meer, Phys. Rev. Lett. 93 (11) (2004) 114802.

[8] B. Steffen, V. Arsov, G. Berden, W. Gillespie, S. Jamison, A.M. MacLeod, A. Van Der Meer, P. Phillips, H. Schlarb, B. Schmidt, et al., Phys. Rev. Spec. Top.-Accel. Beams 12 (3) (2009) 032802.

[9] A.L. Cavalieri, Electro-Optic Characterization of Femtosecond Electron Bunches, 2005.

[10] M. Ferrario, D. Alesini, A. Bacci, M. Bellaveglia, R. Boni, M. Boscolo, P. Calvani, M. Castellano, E. Chiadroni, A. Cianchi, et al., Nucl. Instrum. Methods Phys. Res. Sect. A: Accel. Spectrom. Detectors Assoc. Equip. 637 (1) (2011) S43.

[11] A. Mostacci, M. Bellaveglia, E. Chiadroni, A. Cianchi, M. Ferrario, D. Filippetto, G. Gatti, C. Ronsivalle, Phys. Rev. Spec. Top.-Accel. Beams 15 (8) (2012) 082802.

[12] D. Filippetto, M. Bellaveglia, M. Castellano, E. Chiadroni, L. Cultrera, G. Di Pirro, M. Ferrario, L. Ficcadenti, A. Gallo, G. Gatti, et al., Phys. Rev. Spec. Top.-Accel. Beams 14 (9) (2011) 092804.

[13] R. England, J. Rosenzweig, G. Andonian, P. Musumeci, G. Travish, R. Yoder, Phys. Rev. Spec. Top.-Accel. Beams 8 (1) (2005) 012801.

[14] E. Chiadroni, M. Bellaveglia, P. Calvani, M. Castellano, L. Catani, A. Cianchi, G. Di Pirro, M. Ferrario, G. Gatti, O. Limaj, et al., Rev. Sci. Instrum. 84 (2) (2013) 022703.

[15] E. Chiadroni, A. Bacci, M. Bellaveglia, M. Castellano, G. Di Pirro, M. Ferrario, G. Gatti, E. Pace, A. Rossi, C. Vaccarezza, et al., J. Phys.: Conf. Ser. 359 (2012) 012018 (IOP Publishing). 